\title{
Perfil profissional e sentimento de valorização dos médicos veterinários atuantes na assistência direta aos pacientes no município de Santa Maria/RS/Brasil
}

\author{
The professional profile and feeling of appreciation of veterinarians working directly with \\ assistance to patients in the municipality of Santa Maria/RS/Brazil \\ Perfil profesional y sentimiento de valorización de los médicos veterinarios que actúan en la \\ atención directa de pacientes en la ciudad de Santa Maria/RS/Brasil
}

\section{Resumo}

Médicos veterinários são profissionais da saúde que se dedicam intensamente aos animais que cuidam, executando longas jornadas de trabalho e, por vezes, não tendo seu trabalho valorizado. O município de Santa Maria conta com uma rede de serviços veterinários composta por 2 hospitais, 25 clínicas e um total de 722 profissionais, até esta data, atuando na prevenção e manutenção da saúde dos animais. O objetivo deste estudo é descrever o perfil desses trabalhadores e verificar seu sentimento de valorização profissional. Para isso, foi realizada pesquisa descritiva por meio de questionário aplicado de maneira online, entre os meses de junho e julho de 2021. Participaram médicos veterinários atuantes na assistência direta aos pacientes do município. Entre os 120 profissionais avaliados, 72,5\% eram mulheres, $48,3 \%$ estavam na faixa etária entre 20 e 30 anos, 57,5\% solteiros, 70,9\% exerciam atividades frente ao paciente a menos de 10 anos, 50\% trabalhavam em hospital de instituição pública e 50\% em instituições particulares ou atendimento domiciliar. Desses profissionais, 78,3\% possuíam somente um vínculo empregatício, 49,2\% atuavam com carga horária de trabalho semanal superior a $41 \mathrm{~h}$ e 33,3\% atendiam semanalmente mais de 15 pacientes. Observou-se muitos profissionais com sinais de esgotamento laboral. Dos entrevistados, $70 \%$ não se sentiam valorizados na profissão e $94,2 \%$ consideravam que o médico veterinário não é valorizado no Brasil, porém 62,5\% não gostariam de exercer outra profissão, demonstrando satisfação em exercer a medicina veterinária.

Palavras-chave: Medicina veterinária; Valorização profissional; Perfil dos médicos veterinários.

\begin{abstract}
Veterinarians are health professionals who are intensely dedicated to the animals under their care, performing long working hours and, sometimes, not having their work appreciated. The municipality of Santa Maria has a network of veterinary services composed by 2 hospitals, 25 clinics and a total of 722 professionals up to this date, working to prevent and maintain the health of animals. The objective of this study is to describe the profile of these workers and examine their feeling on professional valorization. For this purpose, a descriptive research was carried out via questionnaire applied online, between June and July of 2021. Veterinarians working directlty with patients in the municipality were subjects of the research. Among the 120 professionals evaluated, $72.5 \%$ were women, $48.3 \%$ were between 20 and 30 years old, $57.5 \%$ were single, $70.9 \%$ had worked directly with the patient for less than 10 years, $50 \%$ worked in a public hospital and 50\% in private institutions or performing home care. Out of these professionals, $78.3 \%$ had only one employment relationship, $49.2 \%$ had a weekly workload of more than 41 hours and $33.3 \%$ saw more than 15 patients per week. There were many professionals with signs of job burnout. Of those interviewed, 70\% did not feel appreciated as professionals and $94.2 \%$ considered that the veterinarian is not appreciated in Brazil, but $62.5 \%$ would not like to practice another profession, demonstrating satisfaction in performing veterinary medicine.
\end{abstract}

Keywords: Veterinary medicine; Professional appreciation; Profile of veterinarians.

\section{Resumen}

Médicos veterinarios son profesionales de la salud que se dedican intensamente a los animales que cuidan, realizando largas jornadas de trabajo y, a veces, sin que se valore su trabajo. El municipio de Santa María cuenta con una red de servicios veterinarios formada por 2 hospitales, 25 clínicas y un total de 722 profesionales, hasta la fecha, trabajando 
para prevenir y mantener la salud de los animales. El objetivo de este estudio es describir el perfil de estos trabajadores y verificar su sentimiento de valorización profesional. Para ello, se realizó una investigación descriptiva a través de un cuestionario aplicado online, entre los meses de junio y julio de 2021. Participaron médicos veterinarios que laboran en la atención directa a los pacientes del municipio. Entre los 120 profesionales evaluados, 72,5\% eran mujeres, 48,3\% tenían entre 20 y 30 años, 57,5\% eran solteros, 70,9\% trabajaban con el paciente a menos de 10 años, $50 \%$ trabajaban en institución hospitalaria pública y $50 \%$ en instituciones privadas o atención domiciliaria. De esos profesionales, $78,3 \%$ poseían una sola relación laboral, 49,2\% trabajaban con carga horaria semanal superior a 41 horas y 33,3\% atendían más de 15 pacientes por semana. Se observó muchos profesionales con signos de agotamiento laboral. De los entrevistados, $70 \%$ no se sentían valorados en la profesión y 94,2\% consideraban que el veterinario no es valorado en Brasil, pero $62,5 \%$ no gustarían de ejercer otra profesión, demostrando satisfacción en el ejercicio de la medicina veterinaria.

Palabras clave: Medicina veterinaria; Valoración profesional; Perfil de los médicos veterinarios.

\section{Introdução}

A abordagem trazida pela medicina veterinária em conjunto com a saúde única incentiva a atuação de forma unificada no Brasil, objetivando atingir saúde de qualidade para as pessoas, ambiente e animais (Cabral \& Mazzucatto, 2017). Neste cenário, os profissionais da saúde merecem cuidados pois são expostos a múltiplos riscos laborais e profundos estímulos emocionais ao tratar de seres doentes em ambientes insalubres (Lundgrén-Laine et al., 2011; Meneghini et al., 2011; Wang et al., 2014). Esses trabalhadores lidam com conflitos e obstáculos diante de cada paciente atendido, podendo gerar impotência, sensação de fracasso, esgotamento, estresse e sentimentos de desvalorização profissional (Shinan-Altman et al., 2016; Urbaneto et al., 2011).

Os médicos veterinários dedicam-se intensamente aos animais de que cuidam, criando empatia por seus tutores e sendo expostos a vários estressores (Zani et al., 2020). Esses profissionais atuam constantemente com eventos traumáticos, dor, sofrimento e morte, sendo submetidos a grande desgaste emocional.

O município de Santa Maria conta com uma rede de serviços veterinários composta por 2 hospitais, 25 clínicas e um total de 722 profissionais, até esta data, atuando na prevenção e manutenção da saúde dos animais. Ainda que este estudo aborde uma pequena amostra do número de profissionais comparada ao total nacional de aproximadamente 160.000 médicos veterinários conforme o último censo (Conselho Federal de Medicina Veterinária, 2020), fica evidente a necessidade de uma atenção especial para esse segmento. Sendo assim, o objetivo deste estudo foi descrever o perfil dos profissionais da área, permitindo conhecer a realidade e verificar seus sentimentos de valorização profissional.

\section{Metodologia}

Trata-se de pesquisa descritiva, com abordagem quantitativa com objetivo de descrever características de determinada população ou fenômeno utilizando técnicas padronizadas de coleta de dados (Gil, 2019). Nesse tipo de pesquisa o pesquisador descreve e registra os fatos, sem interferir neles, conforme proposto por Prodanov e Freitas (2013).

A pesquisa foi realizada através de questionário aplicado de maneira online e direcionado aos médicos veterinários que atuam na assistência direta aos pacientes no município de Santa Maria/RS. Os participantes do estudo constituíram-se de profissionais atuantes tanto na iniciativa privada como em hospital veterinário de instituição pública. Os critérios de inclusão no estudo foram: ser médico veterinário docente, assistente ou pós-graduando e estar atuando na assistência direta aos pacientes do município de Santa Maria/RS. Foram excluídos os trabalhadores que atuam em áreas nas quais não há contato direto com pacientes e/ou que exercem suas atividades profissionais em outros municípios, bem como aqueles que não concordaram com o termo de consentimento (TCLE).

O instrumento online (Quadro 1) foi enviado por e-mail ou aplicativo de mensagens e permaneceu disponível para resposta entre os meses de junho e julho de 2021, sendo obtidas 120 respostas. A análise foi feita através dos recursos do 
Google Forms. Para a efetivação da pesquisa foram seguidos os princípios éticos da Resolução nº66/2012 do Conselho Nacional de Saúde (CNS), sendo aprovada por Comitê de Ética em Pesquisa, sob o número CAAE: 44995021.6.0000.5346.

Quadro 1 - Questionário profissional e sociodemográfico.

\begin{tabular}{|c|c|}
\hline & $\begin{array}{l}\text { Sexo } \\
\text { Feminino ( ) Masculino ( ) }\end{array}$ \\
\hline 2. & $\begin{array}{l}\text { Faixa Etária } \\
20 \text { a } 30 \operatorname{anos}(\text { ) } \quad 31 \text { a } 40 \operatorname{anos}(\text { ) } 41 \text { a } 50 \operatorname{anos}(\text { ) Mais de } 51 \operatorname{anos}(\text { ) }\end{array}$ \\
\hline 3. & $\begin{array}{l}\text { Estado Civil } \\
\text { Solteiro ( ) Casado/união estável ( ) Divorciado/separado ( ) Viúvo ( ) }\end{array}$ \\
\hline 4. & $\begin{array}{l}\text { Marque as alterações que você sentiu nos últimos } 30 \text { dias (marcar quantas opções forem necessárias) } \\
\text { Cefaleia ( ) Insônia ( ) Dores no corpo ( ) Irritabilidade ( ) Cansaço extremo ( ) Dificuldade de concentração ( ) Falta } \\
\text { de apetite ( ) Agressividade ( ) Pessimismo ( ) Problemas de memória ( ) } \\
\text { Quantas horas você costuma dormir? }\end{array}$ \\
\hline 5. & $\begin{array}{l}\text { Faz acompanhamento de saúde mental? } \\
\text { Psicológico ( ) Psiquiátrico ( ) Uso de medicações controladas ( ) Qual? } \\
\text { Não faço nenhum acompanhamento ( ) }\end{array}$ \\
\hline 6. & $\begin{array}{l}\text { Tempo exercido na função (frente paciente) } \\
\text { Inferior a } 1 \text { ano ( ) De } 1 \text { a } 5 \text { anos ( ) De } 6 \text { a } 10 \operatorname{anos}(\text { ) De } 11 \text { a } 15 \operatorname{anos}(\text { ) } \\
\text { De } 16 \text { a } 20 \operatorname{anos}(\text { ) De } 21 \text { a } 25 \operatorname{anos}(\text { ) Acima de } 25 \operatorname{anos}(\text { ) }\end{array}$ \\
\hline 7. & $\begin{array}{l}\text { Local de trabalho } \\
\text { Hospital de instituição pública ( ) Clínica particular ( ) Atendimento domiciliar ( ) }\end{array}$ \\
\hline & $\begin{array}{l}\text { Número de vínculos empregatícios } \\
\text { Um vínculo ( ) Dois vínculos ( ) Mais de dois vínculos ( ) }\end{array}$ \\
\hline & $\begin{array}{l}\text { Carga horária de trabalho semanal (considerando todos os vínculos empregatícios) } \\
\text { De } 1 \mathrm{~h} \text { a } 20 \mathrm{~h}(\mathrm{)} \quad \text { De } 21 \mathrm{~h} \text { a } 30 \mathrm{~h}() \text { De } 31 \mathrm{~h} \mathrm{a} 40 \mathrm{~h}() \quad \text { Mais de } 41 \mathrm{~h}()\end{array}$ \\
\hline & $\begin{array}{l}\text { Quantidade de pacientes que atende diariamente (considerar ambulatoriais, cirúrgicos e internados) } \\
\text { Até } 5 \text { pacientes ( ) De } 6 \text { a } 10 \text { pacientes ( ) Mais de } 10 \text { pacientes ( ) }\end{array}$ \\
\hline & $\begin{array}{l}\text { Sente-se valorizado na profissão? } \\
\operatorname{Sim}(\text { ) Não ( ) }\end{array}$ \\
\hline & $\begin{array}{l}\text { Considera o médico veterinário valorizado no Brasil? } \\
\operatorname{Sim}(\text { ) Não ( ) }\end{array}$ \\
\hline & $\begin{array}{l}\text { Considerando a data atual, gostaria de exercer outra profissão? } \\
\operatorname{Sim}(\text { ) Não ( ) Qual? }\end{array}$ \\
\hline
\end{tabular}

Fonte: Autores.

\section{Resultados e Discussão}

Os resultados e a discussão estão apresentados em quatro categorias: (3.1) caracterização sociodemográfica, (3.2) caracterização profissional, (3.3) caracterização de saúde mental e (3.4) sentimento de valorização profissional.

\subsection{Caracterização sociodemográfica dos médicos veterinários atuantes na assistência direta aos pacientes de Santa Maria/RS}

Os dados referentes ao perfil sociodemográfico dos participantes estão apresentados na tabela 1 . 
Tabela 1 - Caracterização sociodemográfica dos participantes da pesquisa.

\begin{tabular}{|c|c|c|c|}
\hline VARIÁVEL & & FREQUÊNCIA, n & $(\%)$ \\
\hline \multirow{2}{*}{ Gênero } & Feminino & 87 & 72,5 \\
\hline & Masculino & 33 & 27,5 \\
\hline \multirow{4}{*}{ Faixa etária (anos) } & 20 a 30 & 58 & 48,3 \\
\hline & 31 a 40 & 35 & 29,2 \\
\hline & 41 a 50 & 17 & 14,2 \\
\hline & Mais de 51 & 10 & 8,3 \\
\hline \multirow{4}{*}{ Situação conjugal } & Solteiro & 69 & 57,5 \\
\hline & Casado & 46 & 38,3 \\
\hline & Divorciado & 5 & 4,2 \\
\hline & Viúvo & 0 & 0 \\
\hline
\end{tabular}

Fonte: Autores.

Entre os 120 profissionais avaliados, observou-se que as características dos pesquisados demonstrou uma feminização e predominância de faixa etária mais jovem, corroborando outros estudos sobre profissionais de saúde no Brasil (CarrilloGarcía et al., 2013; Costa et al., 2013; Fiúza et al., 2012; Oliveira et al., 2016; Pinto et al., 2010; Tomasi et al., 2008). Acreditase que a parcela maior de participantes solteiros pode estar associada às idades mais jovens e que o número de mulheres reflete o movimento do gênero por igualdade e inserção no mercado de trabalho.

\subsection{Caracterização profissional dos médicos veterinários atuantes na assistência direta aos pacientes de Santa Maria/RS}

Os dados referentes ao perfil profissional dos participantes estão apresentados na tabela 2.

Tabela 2 - Caracterização profissional dos participantes da pesquisa.

\begin{tabular}{|c|c|c|c|}
\hline VARIÁVEL & & FREQUÊNCIA, n & $(\%)$ \\
\hline \multirow{3}{*}{$\begin{array}{l}\text { A) Tempo de trabalho (frente ao } \\
\text { paciente) }\end{array}$} & Menos de 10 anos & 85 & 70,9 \\
\hline & De 11 a 24 anos & 25 & 20,9 \\
\hline & Mais de 25 anos & 10 & 8,2 \\
\hline \multirow{3}{*}{ B) Local de trabalho } & Atendimento domiciliar & 8 & 6,7 \\
\hline & Clínica/hospital particular & 52 & 43,3 \\
\hline & Hospital de instituição pública & 60 & 50 \\
\hline \multirow{2}{*}{$\begin{array}{l}\text { C) Número de vínculos } \\
\text { empregatícios }\end{array}$} & 1 vínculo & 94 & 78,3 \\
\hline & 2 ou mais vínculos & 26 & 21,7 \\
\hline \multirow{3}{*}{$\begin{array}{l}\text { D) Carga horária semanal de } \\
\text { trabalho }\end{array}$} & Até $30 \mathrm{~h}$ & 29 & 24,1 \\
\hline & De $31 \mathrm{~h}$ a $40 \mathrm{~h}$ & 32 & 26,7 \\
\hline & Mais de $41 \mathrm{~h}$ & 59 & 49,2 \\
\hline \multirow{4}{*}{$\begin{array}{l}\text { E) Número de pacientes } \\
\text { atendidos semanalmente }\end{array}$} & Até 5 & 34 & 28,3 \\
\hline & De 6 a 10 & 32 & 26,7 \\
\hline & De 11 a 15 & 14 & 11,7 \\
\hline & Mais de 15 & 40 & 33,3 \\
\hline
\end{tabular}

Fonte: Autores.

No cenário laboral, percebe-se, que há maior representação $(70,9 \%)$ no tempo de trabalho exercido frente ao paciente a menos de 10 anos pelos trabalhadores da área de medicina veterinária. A literatura está em conformidade no que diz respeito aos anos de trabalho, indicando que os profissionais com menos de 10 anos de experiência são a maioria, porém exigem mais 
atenção pois apresentam maior probabilidade de desenvolver estresse ocupacional e sentimentos de desvalorização (Bartram et al., 2009; Hatch et al., 2011; Lovell \& Lee, 2013) (Tabela 2A). Esse resultado vincula-se ao perfil de trabalhadores mais jovens, demonstrado na tabela 1 e acredita-se que está relacionado ao fato de Santa Maria possuir cursos de graduação e pósgraduação em medicina veterinária bastante reconhecidos, atraindo discentes de várias regiões que iniciam suas práticas laborais no município.

Quanto ao local de trabalho (Tabela 2B), 50\% trabalham em hospital de instituição pública e 50\% em instituições particulares ou atendimento domiciliar. Percebe-se que a amostra uniforme entre locais privados e públicos, permitiu comparar as realidades distintas. Acredita-se que este resultado vai de encontro ao esperado, uma vez que o município de Santa Maria conta com uma ampla rede de atendimento em medicina veterinária.

Dos profissionais analisados, torna-se importante ressaltar que 21,7\% possuíam mais de um vínculo empregatício (Tabela 2C). Essa evidência é relatada também por Vasconcelos (2017) numa revisão integrativa sobre profissionais da saúde na qual o autor correlaciona isso à situação financeira do país e remuneração inadequada dos trabalhadores. Deve-se destacar, que a caracterização entre docentes, assistentes e pós-graduandos, não foi considerada para a contabilização de vínculos empregatícios. Essas categorias entrevistadas, pertencem ao quadro clínico-hospitalar de atendimento direto ao paciente, dos distintos estabelecimentos entrevistados. Acredita-se que a baixa remuneração exerce influência no número de vínculos empregatícios e, consequentemente, no sentimento de desvalorização profissional.

$\mathrm{Na}$ variável carga horária (Tabela 2D), 49,2\% dos entrevistados trabalham mais de 41h por semana, corroborando com a afirmação de Silva et al. (2014), Moura et al. (2014) e Muniz et al. (2019) que apontam excesso de trabalho em grande parte dos profissionais da saúde. Esse resultado vai de encontro da assertiva de Souza et al. (2018), que enfatiza em seu estudo a respeito da qualidade de vida dos profissionais atuantes em setores críticos, sobre a redução da jornada de trabalho de até 40 horas ser um aliado para melhoria de vida dessa classe trabalhadora. Acredita-se que esse resultado comprova a necessidade de maior atenção em relação a sobrecarga de trabalho de médicos veterinários, pois certamente impacta na saúde mental e ocupacional desses profissionais.

No que diz respeito ao número de pacientes atendidos semanalmente (Tabela 2E), apenas 33,3\% dos entrevistados atendem mais de 15 pacientes, resultado que difere da pesquisa realizada por Gardner e Hini (2006) com o total de 927 veterinários e determinou que os profissionais que trabalham na assistência direta de animais atendem um elevado número de pacientes, aumentando o risco de sentimento de desvalorização profissional e sobrecarga de trabalho (Hansez et al., 2008). Acredita-se que esse resultado reflete a distribuição uniforme de pacientes na ampla rede de serviços veterinários do município, não havendo número expressivo de atendimentos semanais em uma única instituição ou para um único profissional.

3.3 Caracterização de saúde mental dos médicos veterinários atuantes na assistência direta aos pacientes de Santa Maria/RS

Os dados referentes ao perfil de saúde mental dos participantes estão apresentados na Tabela 3. 
Tabela 3 - Caracterização da saúde mental dos participantes.

\begin{tabular}{|c|c|c|c|}
\hline VARIÁVEL & & FREQUÊNCIA, n & $(\%)$ \\
\hline \multirow{3}{*}{ A) Horas de sono a cada $24 \mathrm{~h}$} & Até $4 \mathrm{~h}$ & 4 & 3,4 \\
\hline & De $5 \mathrm{~h}$ a $7 \mathrm{~h}$ & 85 & 70,8 \\
\hline & Mais de $7 \mathrm{~h}$ & 31 & 25,8 \\
\hline \multirow{10}{*}{$\begin{array}{l}\text { B) Alterações sentidas nos } \\
\text { últimos } 30 \text { dias }\end{array}$} & Cefaleia & 74 & 61,7 \\
\hline & Insônia & 68 & 56,7 \\
\hline & Dores no corpo & 76 & 63,3 \\
\hline & Irritabilidade & 89 & 74,2 \\
\hline & Cansaço extremo & 65 & 54,2 \\
\hline & Dificuldade de concentração & 73 & 60,8 \\
\hline & Falta de apetite & 20 & 16,7 \\
\hline & Agressividade & 36 & 30 \\
\hline & Pessimismo & 67 & 55,8 \\
\hline & Problemas de memória & 77 & 64,2 \\
\hline \multirow{4}{*}{$\begin{array}{l}\text { C) Acompanhamento de saúde } \\
\text { mental }\end{array}$} & Psicológico & 35 & 29,2 \\
\hline & Psiquiátrico & 23 & 19,2 \\
\hline & Uso de medicações controladas & 29 & 24,2 \\
\hline & Não faz acompanhamento & 73 & 60,8 \\
\hline
\end{tabular}

Fonte: Autores.

No que se refere ao sono, 74,2\% dos profissionais dormem menos de $7 \mathrm{~h}$, que é a média de horas de sono recomendada para um adulto saudável restabelecer suas condições orgânicas. Esse resultado reforça que a privação do sono é um fator negativo para a saúde do trabalhador, pois interfere na qualidade de vida e compromete as funções cognitivas e a capacidade para o trabalho, favorecendo a ocorrência de erros (Müller \& Guimarães, 2007; Santos-Coelho, 2020). Sendo assim, acreditase que este resultado merece atenção por parte do próprio profissional, que deve primar por sua saúde e pelo bem-estar dos pacientes aos seus cuidados, procurando melhorar seu sono para não comprometer sua habilidade laboral.

Observou-se muitos profissionais com sinais de esgotamento ocupacional durante a avaliação das respostas dos participantes. Segundo Benevides-Pereira (2002), os portadores de esgotamento profissional apresentam sintomas físicos (cansaço extremo, insônia, dores no corpo, cefaleia); sintomas psíquicos (dificuldade de concentração e alterações de memória) e sintomas comportamentais (irritabilidade, agressividade, pessimismo, falta de apetite). A intensidade e a diversidade dos sintomas dependem de fatores do indivíduo, bem como das condições do ambiente de trabalho (Schaufeli \& Bunnk, 2003) e do sentimento de valorização profissional. Acredita-se que este resultado evidencia a necessidade de acompanhamento de saúde mental regular aos trabalhadores dessa área, para melhorar sua qualidade de vida e evitar doenças ocupacionais.

Entre os participantes, 48,4\% já estão realizando algum tipo de acompanhamento de saúde mental e 24,2\% fazem uso de medicação controlada. Nesse cenário, a medicina veterinária requer maior atenção por apresentar circunstâncias cruciais na incidência de estresse ocupacional e sentimentos de desvalorização. Isso se deve a carga horária elevada, turnos irregulares, problemas com colegas, possíveis reclamações de clientes, acontecimentos inesperados e problemas administrativos ou financeiros (Bartram et al., 2009; Stembert et al., 2003). Este resultado demonstra que ao procurarem ajuda, alguns profissionais já reconheceram a necessidade de buscar melhor qualidade de vida e evitar maiores transtornos de saúde ocupacional. 
3.4 Sentimento de valorização profissional dos médicos veterinários atuantes na assistência direta aos pacientes de Santa Maria/RS

Os dados referentes ao sentimento de valorização profissional dos participantes estão apresentados nas Figuras 1, 2 e 3.

Figura 1 - Você se sente valorizado na profissão?

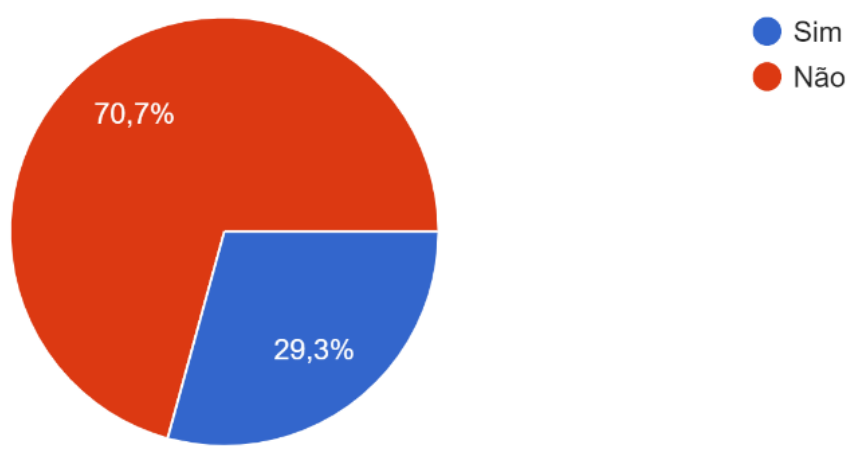

Fonte: Autores.

Figura 2 - Você considera o médico veterinário valorizado no Brasil?

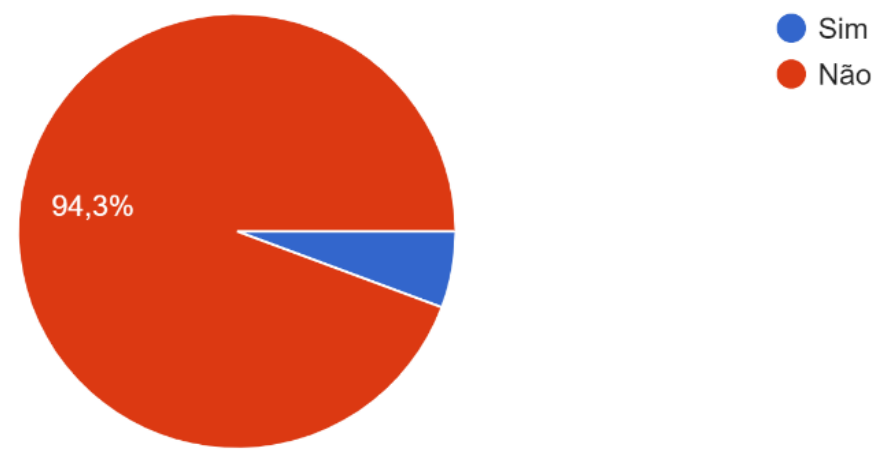

Fonte: Autores.

Figura 3 - Considerando a data atual, você gostaria de exercer outra profissão?

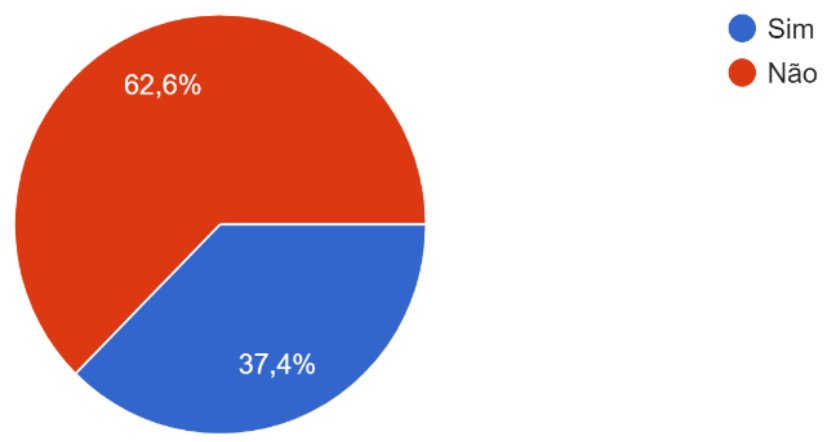

Fonte: Autores.

Vários são os fatores que fazem um profissional se sentir desvalorizado. A falta de estruturação do trabalho, o acúmulo de funções, a sobrecarga de trabalho e a apreciação funcional por outras profissões correlatas são alguns deles, como relata Oliveira (2020). Por outro lado, a necessidade de conseguir uma fonte de renda, mesmo que incompatível com a 
importância do serviço exercido, gera a submissão de profissionais a más condições de trabalho, remetendo à desvalorização da profissão (Oliveira, 2018).

Os resultados deste estudo corroboram os autores acima, pois entre os entrevistados, $70 \%$ não se sentem valorizados na profissão e 94,2\% consideram que o médico veterinário não é valorizado no Brasil. Contudo, 62,5\% não gostariam de exercer outra profissão, demonstrando satisfação em exercer a medicina veterinária. Acredita-se que os profissionais da área experimentam contentamento e bem-estar no atendimento aos seus pacientes, sendo que estes sentimentos se sobressaem em relação aos sentimentos negativos de desvalorização profissional.

Assim, ao resgatar o perfil dos sujeitos deste trabalho e os sentimentos de valorização profissional, percebe-se que, na contramão do descontentamento relatado, está a satisfação relacionada ao trato direto com os animais. Isso é percebido quando a maioria dos participantes não deseja mudar de profissão. Ainda que o questionário tenha sido aplicado ao longo da pandemia de COVID-19 (junho e julho de 2021), os autores entendem que as respostam não sofreram influência direta dessa situação. Em linhas gerais, o questionário foi direcionado para reflexão sobre os anos de profissão e situações relacionadas com a trajetória profissional de cada participante.

\section{Conclusão}

Esta pesquisa identificou o perfil e o sentimento de valorização profissional de médicos veterinários atuantes na assistência direta aos pacientes do município de Santa Maria/RS. Observou-se que os profissionais são na maioria mulheres, entre 20 a 30 anos de idade, solteiras, com um vínculo empregatício e carga horária de trabalho superior a 41h semanais.

Uma parte considerável desses trabalhadores não se sente reconhecido e considera que os médicos veterinários não são valorizados no Brasil. Porém, a maioria não gostaria de exercer outra profissão. Acredita-se que a constatação do sentimento de pouca valorização profissional aponta para a necessidade de olhar mais atento no sentido de que estratégias sejam implementadas levando à melhoria da qualidade de vida laboral e diminuindo o risco de adoecimento relacionado ao trabalho.

\section{Agradecimentos}

À Coordenação de Aperfeiçoamento de Pessoal de Nível Superior (CAPES) e ao Conselho Nacional de Desenvolvimento Científico e Tecnológico (CNPQ).

\section{Referências}

Bartram, D. J., Yadegarfar, G. \& Baldwin, D. S. (2009). Psychosocial working conditions and work-related stressors among UK veterinary surgeons. Occupational Medicine, 59(5), 334-341. https://doi.org/10.1093/occmed/kqp072

Benevides-Pereira, A. M. T. (2002). Burnout: quando o trabalho ameaça o bem-estar do trabalhador. Casa do Psicólogo.

Cabral, A. P. M. \& Mazzucatto, B. C. (2017). Avaliação do conhecimento dos tutores de cães e gatos atendidos no hospital veterinário-UEM Umuarama sobre a importância da temática: saúde oral. Revista de Ciência Veterinária e Saúde Pública, 4, 34-34. https://doi.org/10.4025/revcivet.v4i0.37094

Carrillo-García, C., Solano-Ruíz, M. del C., Martínez-Roche, M. E. \& Gómez-García, C. I. (2013). Influência do gênero e da idade: satisfação no trabalho de profissionais da saúde. Revista Latino-Americana de Enfermagem, 21(6), 1314-1320. https://doi.org/10.1590/0104-1169.3224.2369

Conselho Federal de Medicina Veterinária (2020). Censo. https://www.cfmv.gov.br/censo/transparencia/2017-2020/2020/12/11/

Costa, S. M., Prado, M. C. M., Andrade, T. N., Araújo, E. P. P., Silva Júnior, W. S., Gomes Filho, Z. C. \& Rodrigues, C. A. Q. (2013). Perfil do profissional de nível superior nas equipes da Estratégia Saúde da Família em Montes Claros, Minas Gerais, Brasil. Revista Brasileira de Medicina de Família e Comunidade, 8(27), 90-96. http://dx.doi.org/10.5712/rbmfc8(27)530

Fiúza, T. M., Ribeiro, M. T. A. M., Gomes, K. W. L., Pequeno, M. L., Barreto, I. C. H. C., Miranda, A. S., Oliveira, P. R. S. \& Montenegro Júnior, R. M. (2012). Necessidades educacionais dos profissionais da Estratégia Saúde da Família (ESF): possibilidades de Educação em Saúde no município de Fortaleza (CE). Revista Brasileira de Medicina de Família e Comunidade, 7(24), 139-146. https://doi.org/10.5712/rbmfc7(24)186 
Gardner, D. H. \& Hini, D. (2006). Work-related stress in the veterinary profession in New Zealand. New Zealand Veterinary Journal, 54(3), 119-124. https://doi.org/10.1080/00480169.2006.36623

Gil, A. C. (2019). Métodos e técnicas de pesquisa social (7a. ed., v. 1). Atlas.

Hansez, I., Schins, F. \& Rollin, F. (2008). Occupational stress, work-home interference and burnout among Belgian veterinary practitioners. Irish Veterinary Journal, 61(4), 1-9. https://link.springer.com/article/10.1186/2046-0481-61-4-233.

Hatch, P. H., Winefield, H. R., Christie, B. A. \& Lievaart, J. J. (2011). Workplace stress, mental health, and burnout of veterinarians in Australia. Australian Veterinary Journal, 89(11), 460-468. https://doi.org/10.1111/j.1751-0813.2011.00833.x

Lovell, B. L. \& Lee, R. T. (2013). Burnout and health promotion in veterinary medicine. The Canadian Veterinary Journal, 54(8), 790-791. https://www.ncbi.nlm.nih.gov/pmc/articles/PMC3711171/

Lundgrén-Laine, H., Kontio, E., Perttilä, J., Korvenranta, H., Forsström, J. \& Salanterä, S. (2011). Managing daily intensive care activities: an observational study concerning ad hoc decision making of charge nurses and intensivists. Critical Care, 15(4), R188. https://link.springer.com/article/10.1186/cc10341

Meneghini, F., Paz, A. A. \& Lautert, L. (2011). Occupational factors related to burnout syndrome components among nursing personnel. Texto \& Contexto Enfermagem, 20(2), 225-233. https://www.scielo.br/pdf/tce/v20n2/a02v20n2.pdf

Moura, R. S., Reis, R. P., Melo, E. S., Maranhão, I. M. \& Alécio, M. S. (2014). Estresse da equipe de enfermagem atuante no cuidado na UTI adulto: Revisão integrativa. Revista Hórus, 9(1), 35-52. https://www.researchgate.net/publication/274082042

Müller, M. R. \& Guimarães, S. S. (2007). Impacto dos transtornos do sono sobre o funcionamento diário e a qualidade de vida. Estudos de psicologia, 24(4), 519-528. https://doi.org/10.1590/S0103-166X2007000400011

Muniz, D. C., Andrade, E. G. S. \& Santos, W. L. (2019). A saúde do enfermeiro com a sobrecarga de trabalho. Revista de Iniciação Científica e Extensão, 2(Esp. 2), 274-279. https://revistasfacesa.senaaires.com.br/index.php/iniciacao-cientifica/article/view/275

Oliveira, A. L. (2018). O desemprego dos Jovens [Trabalho de Conclusão de Curso em Ciências Econômicas, Universidade de Brasília]. https://bdm.unb.br/handle/10483/24489

Oliveira, D. C. B. S. (2020). O papel do Médico Veterinário na Saúde Pública e sua visibilidade como profissional de saúde: experiência e reflexões [Monografia de Especialização em Saúde Pública, Escola de Saúde Pública do Estado de Minas Gerais]. http://repositorio.esp.mg.gov.br:8080/xmlui/bitstream/handle/123456789/356/TCC\%20Daniela\%20Carla\%20Bernardes\%20Silva\%20de\%20Oliveira\%20.pdf? sequence $=1 \&$ is Allowed $=$

Oliveira, M. P. R., Menezes, I. H. C. F., Sousa, L. M. \& Peixoto, M. R. G. (2016). Formação e Qualificação de Profissionais de Saúde: Fatores Associados à Qualidade da Atenção Primária. Rev. bras. educ. med., 40(4), 547-559. https://doi.org/10.1590/1981-52712015v40n4e02492014

Pinto, E. S. G., Menezes, R. M. P. \& Villa, T. C. S. (2010). Situação de trabalho dos profissionais da Estratégia Saúde da Família em Ceará-Mirim. Rev Esc Enferm USP, 44(3), 657-664. http://www.scielo.br/pdf/reeusp/v44n3/15.pdf

Prodanov, C. C. \& Freitas, E. C. (2013). Metodologia do Trabalho Científico: Métodos e Técnicas da Pesquisa e do Trabalho Acadêmico (2a ed.). Freevale.

Santos-Coelho, F. M. (2020). Impacto da privação de sono sobre cérebro, comportamento e emoções. Medicina Interna de México, 36(S1), 17-19. https://www.medigraphic.com/pdfs/medintmex/mim-2020/mims201f.pdf

Schaufeli, W. B. \& Buunk, B. P. (2003). Burnout: An overview of 25 years of research and theorizing. In M. J. Schabracq, J. A. M. Winnubst \& C. L. Cooper, The Handbook of Work and Health Psychology (pp. 383-425). John Wiley \& Sons Ltd.

Shinan-Altman, S., Werner, P. \& Cohen, M. (2016). The connection between illness representations of Alzheimer's disease and burnout among social workersand nurses in nursing homes and hospitals: a mixed-methods investigation. Aging \& mental health, $20(4), 352-361$. https://doi.org/10.1080/13607863.2015.1008983

Silva, G. K. C., Avelino, F. V. S. D., Gouveia, M. T. O., Madeira, M. Z. A. \& Avelino, F. P. D. (2014). Síndrome de Burnout em enfermeiros atuantes em unidade de terapia intensiva. Enfermagem Foco, 5(3/4), 75-78. https://doi.org/10.21675/2357-707X.2014.v5.n3/4.563

Souza, V. S., Silva, D. S., Lima, L. V., Teston, E. F., Benedetti, G. M. S., Costa, M. A. R., \& Mendonça, R. R. (2018). Qualidade de vida dos profissionais de enfermagem atuantes em setores críticos. Revista Cuidarte, 9(2), p. 2177-2186. https://doi.org/10.15649/cuidarte.v9i2.506

Stembert, F. M., Lipman, L. J. \& Loomans J. B. (2003). Veterinarian: a healthy profession?. Tijdschrift Voor Diergeneeskunde, 128(18), 565-569. https://europepmc.org/article/med/14535074

Tomasi, E., Facchini, L. A., Piccini, R. X., Thumé, E., Silveira, D. S., Siqueira, F. V., Rodrigues, M. A., Paniz, V. V. \& Teixeira, V. A. (2008). Perfil sóciodemográfico e epidemiológico dos trabalhadores da atenção básica à saúde nas regiões Sul e Nordeste do Brasil. Cadernos de Saúde Pública, 24(1), s193s201. http://www.scielo.br/pdf/csp/v24s1/23.pdf

Urbanetto, J. S., Silva, P. C., Hoffmeister, E., Negri, B. S., Costa, B. E. P., \& Figueiredo, C. E. P. (2011). Workplace stre ss in nursing workers from na emergency hospital: Job Stress Scale analysis. Revista latino-americana de enfermagem, 19(5), 1122-1131. https://doi.org/10.1590/S010411692011000500009

Vasconcelos, M. L. (2017). Síndrome de Burnout em enfermeiros que trabalham em unidades de terapia intensiva: Revisão integrativa [Trabalho de Conclusão de Curso em Enfermagem, Faculdade de Ciências da Saúde, Universidade de Brasília]. https://bdm.unb.br/handle/10483/20086 
Research, Society and Development, v. 11, n. 3, e9411326161, 2022

(CC BY 4.0) | ISSN 2525-3409 | DOI: http://dx.doi.org/10.33448/rsd-v11i3.26161

Wang, W., Lopez, V., Chow, A., Chan, S. W. C., Cheng, K. K. F., \& He, H. G. (2014). A randomized controlled trial of the effectiveness of a self-help psychoeducation programme on outcomes of outpatients with coronary heart disease: study protocol. Journal of Advanced Nursing, 70(12), 2932-2941. https://doi.org/10.1111/jan.12397

Zani, G. L., Rosa, C. L. \& Machado, M. A. (2020). Sindrome de Burnout e a fadiga da compaixão: das vulnerabilidades dos profissonais de veterinária/Burnout's syndrome and the fatigue of compassion: the vulnerabilities of veterinary professionals. Brazilian Journal of Development, 6(1), 41074123. https://doi.org/10.34117/bjdv6n1-292 